\title{
Digital Assembly Process Planning Model of Frame Subassembly for General Aircraft
}

\author{
Jiangyan JIN ${ }^{1, a^{*} \text {, Lanzhi GU }}{ }^{2, b}$, Yixiang FANG ${ }^{1, c}$ and Hairu YIN ${ }^{1, d}$ \\ ${ }^{1}$ School of Mechanical Engineering, Hebei University of Science \& Technology, 050000, Hebei \\ Shijiazhuang ,China \\ ${ }^{2}$ Hebei Chemical and Pharmaceutical College, 050026, Hebei Shijiazhuang, China \\ ajinjy1108@163.com, b gulanzhi1971@126.com, 'fang_yixiang@163.com, d438031465@qq.com
}

Keywords: General aircraft; Assembly; Process planning; Digital model; Simulation

Abstract. Traditional transfer mode of process data cannot meet the needs of modern general aircraft. Digital data transfer is basis for digital aircraft assembly. In view of actual enterprise requirements for digital process planning, a frame subassembly is taken into study object, a 3D visual assembly process planning model is proposed. Through analyzing aircraft manufacturing process planning, process information can be divided into three types: product, process, and resource. In the model, detailed contents of the three and internal relationship among product structure, process planning and resources are represented. According to the model, the process is the core, the product and related resources are closely connected with the process nodes. Finally, the model is constructed and related process simulation is carried out on DELMIA platform, therefore digital process data delivery is realized.

\section{Introduction}

Assembly is a vital wok in the whole process of design and manufacturing of aircraft [1]. Assembly process planning is a technology preparation for aircraft assembly, in which designer works out process plan according to design requirements. And process data to guide actual production is obtained. Traditionally, the process data exist as text and entity, such as process files, data and tools in kind, which does not meet the "low cost, short cycle, and good quality" demands of aircraft manufacturing. However, in order to meet the requirements for aircraft manufacturing, it is the most effective to establish digital process data transfer mechanism based on 3D solid model and digital inspection technology [1,2].

Some large enterprises, such as Boeing, have established digital assembly process design and simulation system based on 3D assembly process planning model [3]. In developing Aviation industry enterprises, 3D process design and assembly simulation has been only performed at local level, and 3D process plan has not been obtained and transferred to terminal operator [4-6]. In general aviation enterprises, it is a particularly case.

Through analyzing assembly process planning of a frame subassembly for general aircraft, a variety of process information is put in order in digital environment to construct an assembly process planning model. Based on the model, digital assembly process planning and simulation for frame subassembly is proposed.

\section{Assembly Process Design for Aircraft}

At present, aircraft assembly process design can be described as shown in Fig. 1. Process planning begins after proper design data has been received. At first, in view of the actual production condition, process separation faces are defined and assembly unit partition is performed. Then detailed process design begins, including defining assembly datum, working out coordination scheme, planning assembly sequence, determining processes and steps, selecting or designing tools and so on. Detailed process plan should be verified by assembly process simulation. If some question is found, process plan must be not modified until satisfied simulation is gotten. Finally, 3D assembly order can be given to guide shop production. 


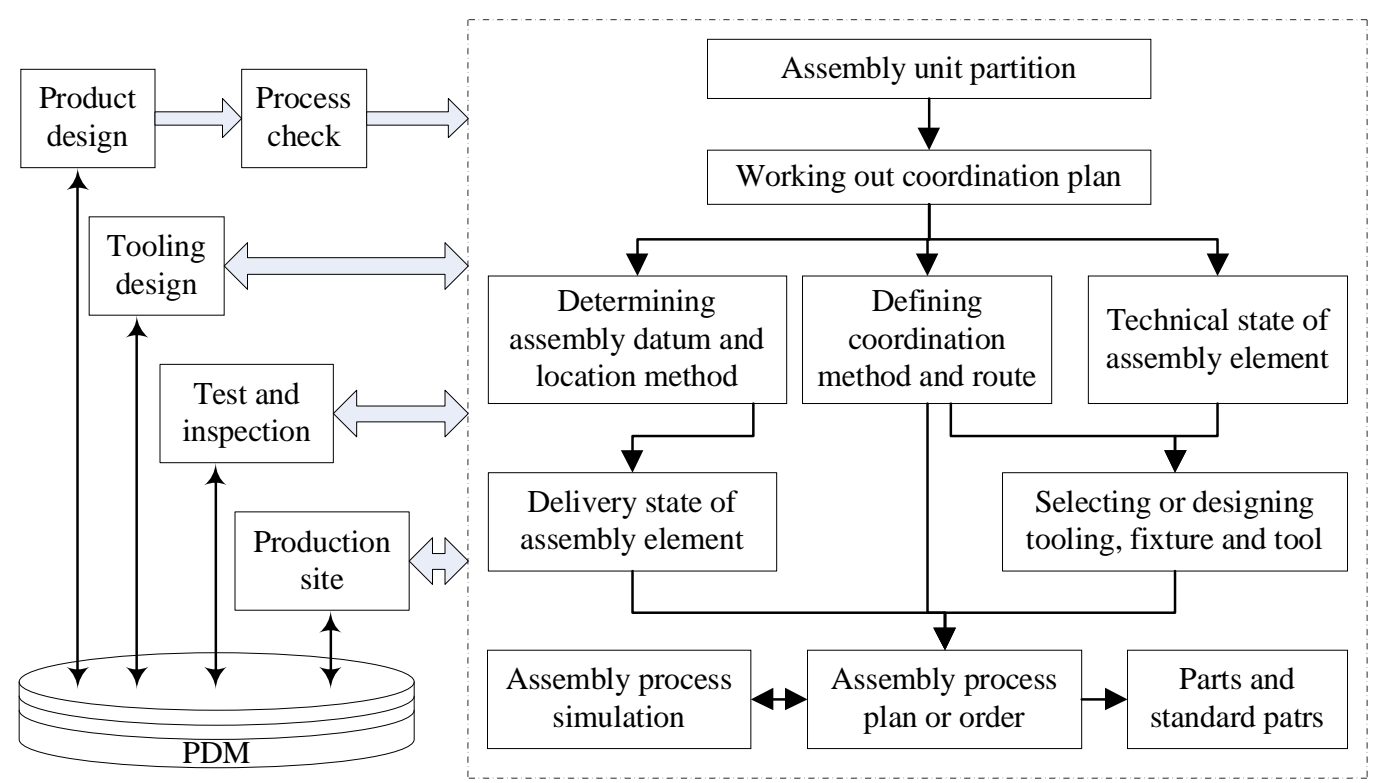

Fig. 1 Aircraft assembly process design process

A model of a frame subassembly of general aircraft is shown in Fig. 2, which consists of diaphragms, webs, internal and external architraves, liners, pedestals, angle cleats. It has thirty four parts.

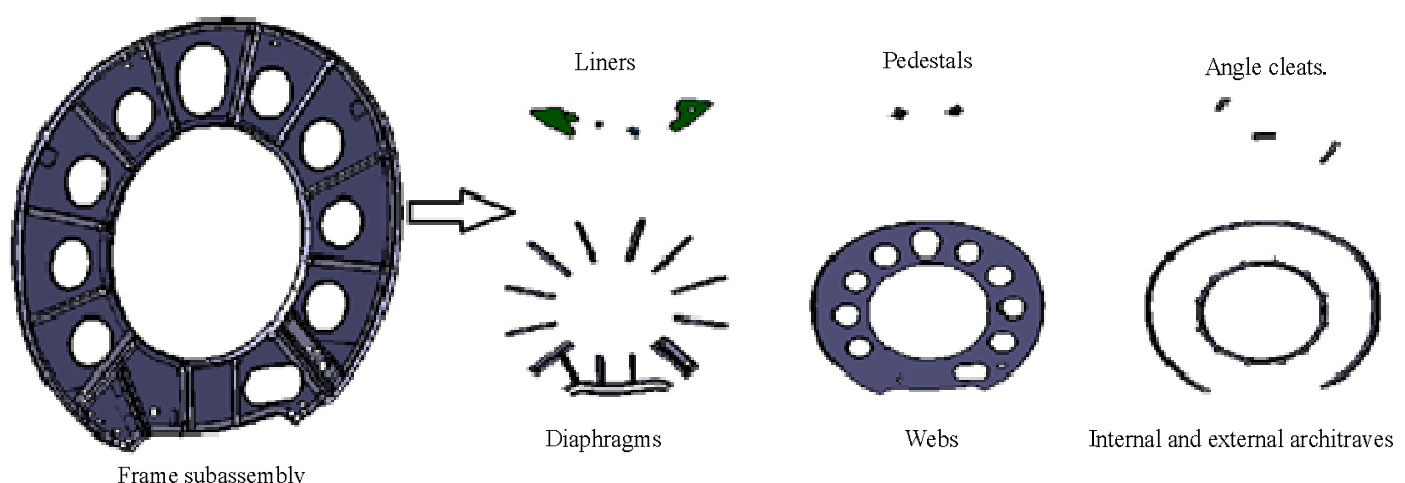

Fig. 2 Frame subassembly

Frame subassembly is an assembly unit, its assembly process is describes below.

(1) Determining location datum and mode

ü Location data for architraves are locating holes in assembly tooling and graduating line for seaming, and architraves are located with bolt pins.

ü Location data for web are assembly holes, and webs are located with bolt pins.

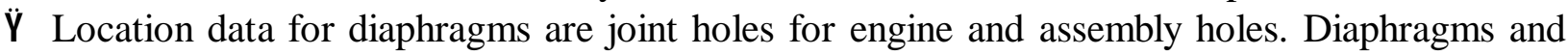
webs are located with bolt pins.

ü Location data for liners are assembly holes of the web, and liners are located with marking and drilling.

ü Location data for pedestals are assembly holes of the web, and pedestals are located with spring pins.

(2) According to location datum and mode, Locating and clamping all the parts are performed on the assembly tooling in proper assembly sequence, that is, internal architrave, web, external architrave, diaphragms, angle cleats, liner and pedestals.

(3) Drilling holes and riveting all the parts.

\section{Assembly Process Planning Model for General Aircraft}

To generate digital process data, it is very important to represent various process information and relationship among them.

Process Information Constitution. Assembly process planning information can be collected after 
analyzing assembly process of typical component, which include product model, tooling model, process plan and other process attributes. Assembly process information model can be represented as Eq. 1:

$$
M=P_{\mathrm{d}} \cup P \cup R
$$

In Eq. $1, P_{\mathrm{d}}$ indicates 3D product model. $P$ indicates all process plan corresponding to product design model. $R$ indicates 3D Tooling model and other tools for assembly.

(1) Product model $P_{\mathrm{d}}$ includes 3D geometry and structure of all the parts and standard parts, and variety of attributes, such as parts code, name, number, weight, and material. It is sole source for assembly process planning, and a basis for assembly process simulation.

(2) Process plan information $P$ is derived from assembly process planning. It describes assembly unit partition, assembly sequence and process attributes. It is a hierarchical model and can be described below:

$$
P=\left\{N_{\mathrm{p}}, R_{\mathrm{s}}, A\right\} .
$$

In Eq. 2, $N_{\mathrm{p}}=\left\{n_{11}, n_{21}, \mathrm{~L} n_{2 x}, \mathrm{~L}, n_{m 1}, \mathrm{~L}, n_{m n}\right\}$ expresses all process nodes, $n_{i j}$ means the jth assembly unit on the ith layer. $m$ is the number of layer. $n$ is the number of units on the mth layer. $R_{\mathrm{s}}$ is a set of relationships of affiliation between two units. $R_{\mathrm{s}}=\{\langle f, c\rangle \mid f, c \in P\}, f$ is parent node of $c \circ A$ is a set of process attributes including node number, name, edition, task description, assembly datum, test demands, delivery status and so on.

(3) $R$ represents manufacturing resource information, such as 3D model of tooings, locating and clamping methods, assembly tool information, name, number, quantity, designers and operator. Each assembly tooling is corresponding with a process node, but not all process nodes are corresponding with the toolings.

Process Planning Modeling. In traditional assembly process design, there is less relevance between process information, and all process information are presented by written language Product model, tooling information and process plan are segregated. In digital process design, all process dimensions and tolerances are attached to 3D product model, and the relevance among product model, process plan and tooling information can be established. Through analyzing all the information involved in process design and the relationship between $3 \mathrm{D}$ model and process information, the assembly process planning model for the frame subassembly is constructed, as shown in Fig. 3.

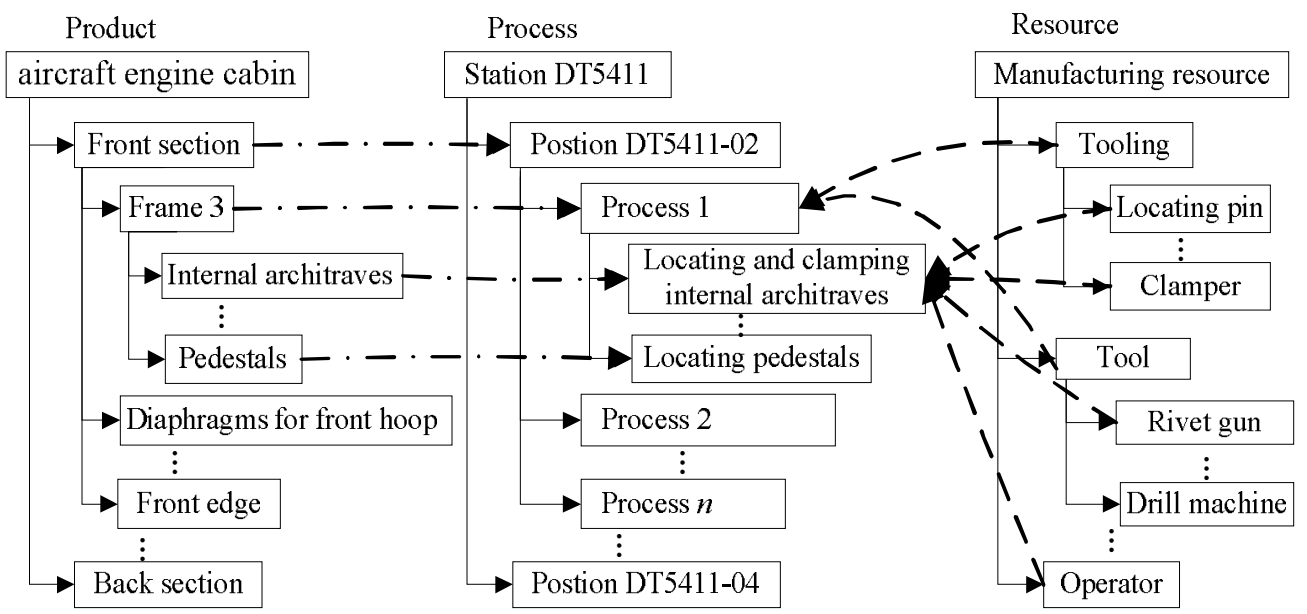

Fig. 3 Assembly process planning model

In the assembly process planning model, product is the basis, process is the core, and resource is the assistant. They are indispensable. A step is the minimum unit of process, and each step is corresponding to each part of product and relevant closely to assembly tool, for example, riveter and the like. A process, which is usually carried out with assembly tooling, consists of several steps. A position 
includes one process or several processes. A station is made up of several positions. In addition, on every process node, variety of process attributes, such as name, datum, accuracy and operating demands, are described. Thus, the parts are designated to process nodes, and the tooling and tools are matched with relevant process nodes. The internal relationships among product, process, and resource are constructed. The model is integrated data set for assembly process simulation.

\section{Digital process planning and simulation}

In digital process planning, based on the 3D product model, process designers construct digital assembly process planning model. After verified with simulation, feasible and reasonable assembly process is worked out. Then process diagrams and operating videos for all process nodes are presented to draw up 3D visual process plan. Simulation content of different process design stage is different. In the process evaluation phase, simulation is carried out to analyze the assemblability of assembly units consisted of parts and components, and to check the rationality of structure design. In the process preparing phase, assembly unit partition, assembly data, assembly sequence and other process information are determined, and applications for tooling are submitted. In the tooling design phase, qualified toolings are design. In detailed process planning, according to assembly process planning model, product, process and resource and the relevance among the three are modeled, process simulation is carried out and operating videos are obtained.

DELMIA (Digital Enterprise Lean Manufacturing Interactive Application) is digital software for planning manufacturing process, process simulation, human-machine engineering and so on. On DELMIA platform, the modeling process can be described below:

At first, establishing product design tree, assembly process tree and resource tree. It includes three steps: (1) importing 3D product model and corresponding product unit partition; (2) the resource is classified into tooling, fixture, tool, and operator, and 3D models of some resources are imported; (3) making process partition and establishing separately various process nodes: station, position, process and step. For every process node, assembly hierarchy and sequence is defined and assembly tasks are set up. Detailed process information, such as location datum, locating way and operating method, are designated. Then relevance among product, process and resource is modeled. Digital assembly process planning process and outcome for frame subassembly is shown in Fig. 4.

After working out the assembly process plans, all the assembly hierarchy, sequence and task must be verified by assembly process simulation. Simulation should be made in layers from child nodes to parent nodes. Namely, step sequence is firstly simulated in a process node, and then process sequence is simulated in a position, and so on, all assembly simulations are performed. In simulation process, assembly path of parts can be planned and assembling interference between parts and between parts and tooling can be seen. With assembly simulation, unreasonable process plan is found. Assembly process planning model must be not continuously modified and improved until reasonable process plan is gotten. Assembly simulation process is shown in figure 5, which include assembly sequence, path and interference detection. Assembly simulation of every part or component correspond one to one with process node and resource node. Combining assembly process planning model and simulation video can realize $3 \mathrm{D}$ visual process design and application.

\section{Conclusion}

In this paper, through analyzing assembly process of a frame subassembly in detail, an assembly process planning model is constructed. According to the model, detailed content of product, process and resource is represented and the relationship of the three is defined. Based on the model, digital process planning and simulation is realized, and thus the digital process data can be delivered to production site. Therefore, increases in the production rate, reduction in production cost and time are achieved. 


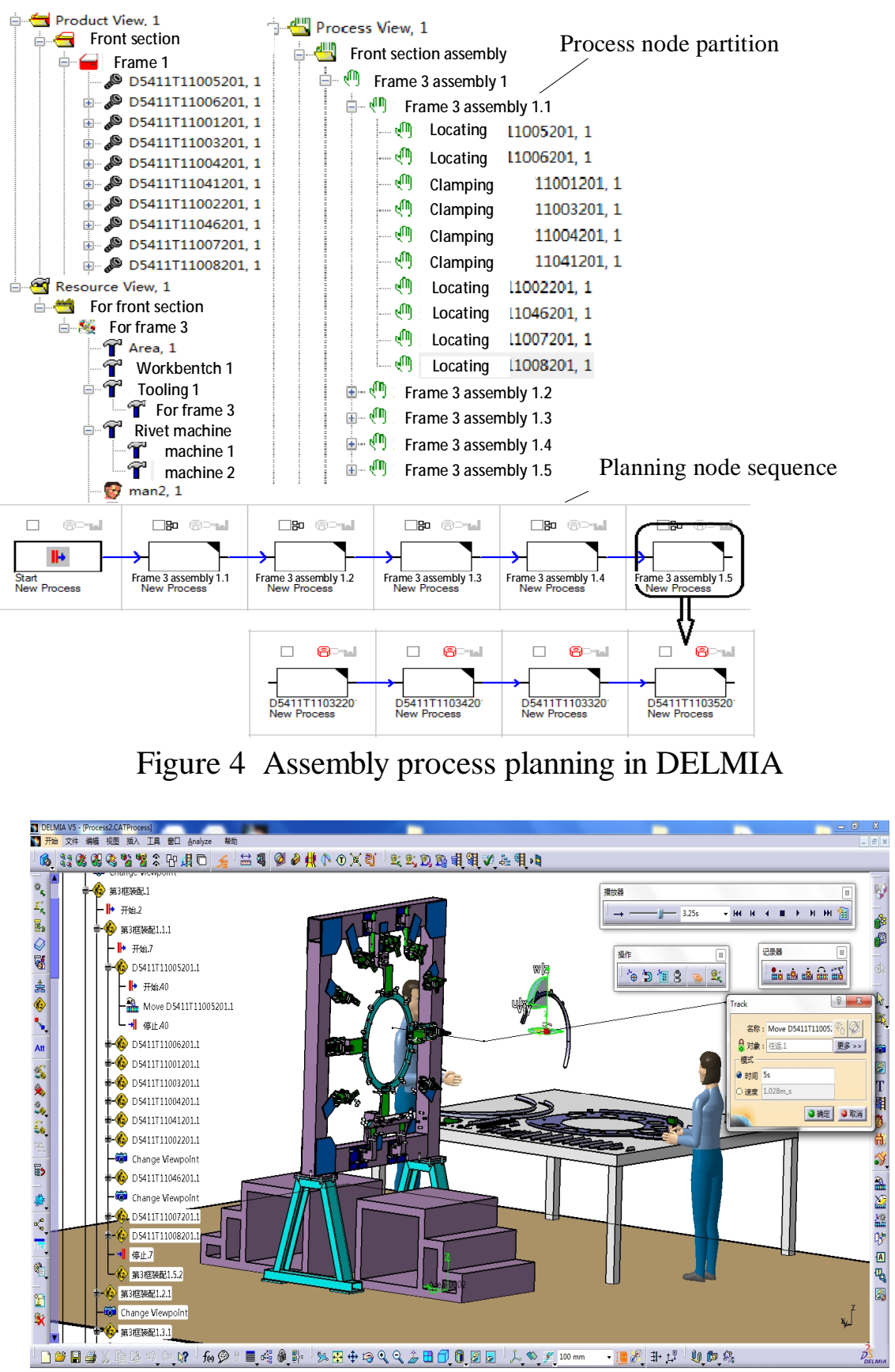

Figure 5 Assembly process simulation

\section{Acknowledgements}

The work is supported by the Hebei Provincial Major Basic Research Program, China (No. 14961811D), the University and College Science and Technology Research Project in Hebei Province, China (No. QN2014207), the Science Foundation of Hebei University of Science and Technology, China (No. TY01, No. TY27). The authors are also grateful to the editors and the anonymous reviewers for helpful comments.

\section{References}

[1] Chief Compiling Committee of Aeronautic Manufacturing Engineering Manual. Aeronautic manufacturing engineering manual: aircraft assembly. Aviation Industry Press, Beijing, 2010.

[2] Y.Q. Fan, Modern aircraft manufacturing technology, Beijing University of Aeronautics and Astronautics Press, Beijing, 2001. 
[3] Z.Q. Yu, S. Chen, W. Sun, et al, Application of MBD-Based Three-Dimensional Module in Aircraft Manufacturing, Aeronautical Manufacturing Technology, 25 (2009), 82-85.

[4] W. Luo, B.S. Tong, Model Based Technology of Aircraft Process Planning, Verification and Execution, Aeronautical Manufacturing Technology, 18 (2010), 73-76.

[5] T.T. Feng, X. Jin, M. Wang, et al, MBD-Based Model Design for Aircraft Assembly Process, Aeronautical Manufacturing Technology, 24 (2010), 95-98.

[6] J.T. Guo, Z.Y. Mei, Design and Application of MBD-Based Aircraft Digital Assembly Process, Aeronautical Manufacturing Technology, 22 (2011), 74-77. 